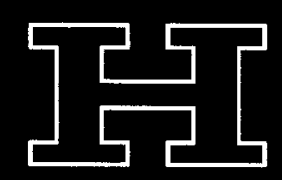

DD
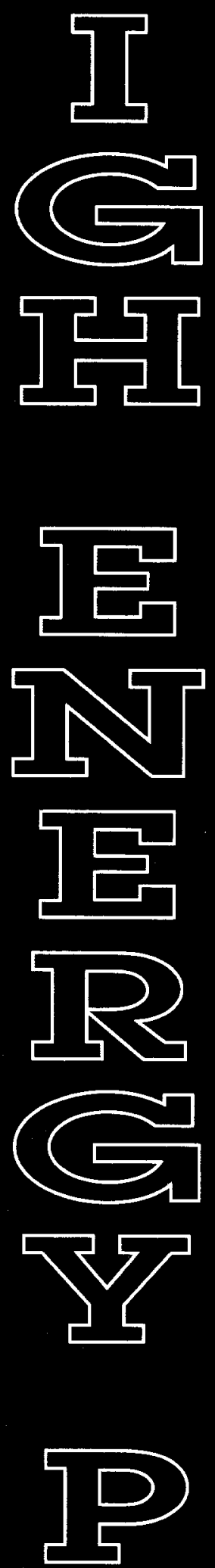
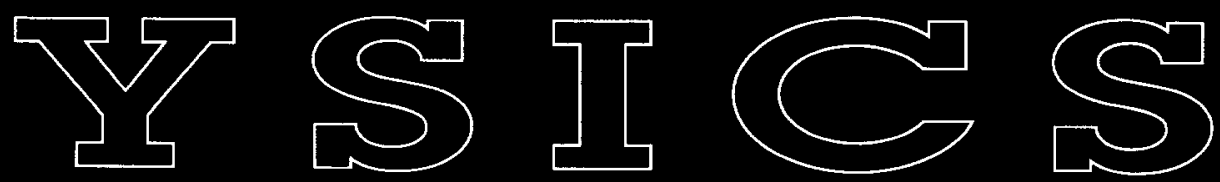


\title{
A Precision Technique for Mounting Scintillating Fiber Ribbons for Charged Particle Tracking
}

\author{
S. Carabello, D. Gau, B. Howell, D.Koltick and Yu. Pischalnikov \\ Purdue University, West Lafayette, IN 47906 \\ D. Michael, \\ FEPCO Coordinate Measuring Services, Noblesville, IN 46060
}

\begin{abstract}
We have undertaken a research program to develop a Scintillating Fiber charged particle Tracking (SFT) detector for the D0 upgrade experiment at FNAL. We have developed a construction method utilizing scintillating fibers first accurately formed into ribbons, then precisely locating these ribbons on the inside and outside of a lightweight cylindrical base. A Coordinate Measuring Machine (CMM) is used to control each step of the ribbon mounting procedure. Ribbons $2 \mathrm{~m}$ long, containing 255 fibers each have been placed on composite structures with accuracy $\sim 20 \mu \mathrm{m}$. The technique for producing highly accurate fiber ribbons and the method of precision placement of ribbons are presented. The spatial calibration of a charged particle tracker using the CMM measurements are discussed.
\end{abstract}

\section{INTRODUCTION}

In future collider experiments, such as the upgraded $D 0$ and $C D F$ detectors, the advantages of low occupancy rates and fast response time make scintillation fibers an excellent choice for a charged particle tracker[1]. However one is confronted with the problem of how to position fibers accurately for good spatial resolution. Fiber positioning is complicated by the fact that the fibers cannot be significantly stressed without being damaged and the large number of readout channels that must be accommodated $(-80,000$ in the case of D0). Because of this many degrees of freedom must be removed when precisely fixing each fiber's location. The ribbon concept is a modular approach to this problem. The modularity of ribbons allows the handling of many channels at one time and greatly reduces the number of degrees of freedom to just a few per ribbon. With this approach we have developed a construction method whereby precisely made fiber ribbons can be mounted on an accurate, lightweight, composite base while at the same time the spatial calibration is completely determined during the construction process.

\section{SCINTILLATING FIBER RIBBON}

\section{A. Fiber Ribbon Construction}

The basic measuring element of our tracker consists of $830 \mu \mathrm{m}$ diameter multiclad polystyrene scintillating fibers. These fibers are formed into a doublet ribbon containing two parallel layers of fibers. The construction technique employees a high precision v-grooved hard alloy 7075 aluminum jig-plate $3 \mathrm{~m}$ in length, with groove's straight and parallel to $\sigma=10 \mu \mathrm{m}$ (Figure 1). To make the ribbon, fibers are first placed in the grooves in the jig plate to form the lower layer of the ribbon doublet. The upper layer is then added by placing fibers in the recesses between the fibers in the lower layer. This insures a complete correlation between two layers. The ribbons exhibit local rigidity, yet global flexibility, due to simple water-based adhesives that are used.

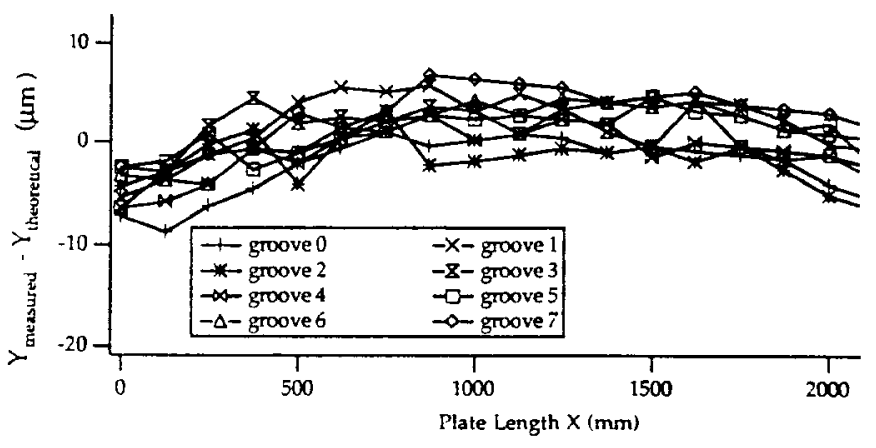

Fig. 1 Qualification of ribbon production jig-plate.

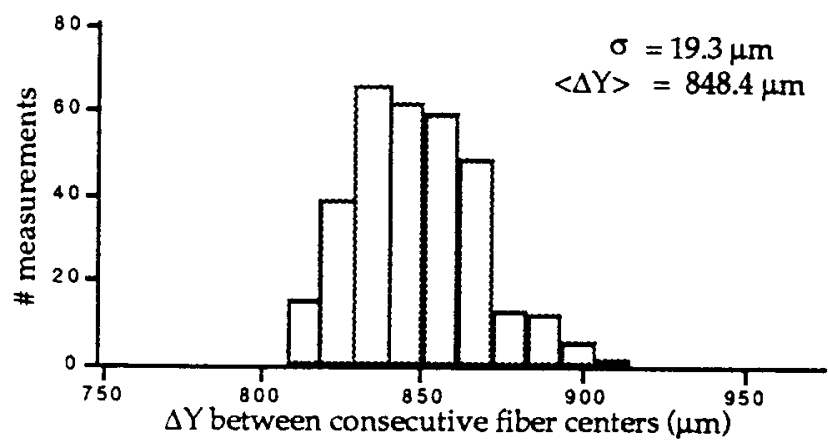

Fig. 2 The spacing $\Delta Y$ between adjacent fiber centers in the upper singlet of the test ribbon.

Measurements of the latice spacing, performed with the coordinate measuring machine, show that the structure is consistent to $\sigma<20 \mu \mathrm{m}$ (Figure 2). The ribbons contained 255 fiber elements.

\section{B. Goals for Ribbons Placement Accuracy}

Accuracy requirements for fiber placement are driven by need for good spatial resolution for charged particle tracking 
without excessive calibration. The estimated doublet resolution is then given by:

$$
\sigma_{\text {doublet }}^{2} \equiv \sigma_{\text {geometry }}^{2}+\sigma_{\text {alignment }}^{2} \text {, }
$$

where $\sigma_{\text {geometry }}^{2}=\mathrm{D} / 2^{*}(12)^{-0.5}$ is the limiting resolution of tightly-spaced doublet ribbon geometry with fibers of finite fiber diameter $D$, and $\sigma_{\text {alignment }}$ is the contribution due to misalignment of placing the ribbon onto the surface of the composite structure. For the case of prototype tests, $\mathrm{D}=850 \mu \mathrm{m}$ which yields $\sigma_{\text {geometry }} \cong 125 \mu \mathrm{m}$. If we make a superlayer where doublets are offset by exactly $1 / 4$ the fiber spacing, the $\sigma_{\text {geometry }}$ is improved by a factor of 2 to -60 $\mu \mathrm{m}$. To achieve the best possible resolution the ribbons must be placed with an accuracy uncertainty that is less than this. Our construction goals for placement accuracy are $\sigma_{\text {alignment }}<50 \mu \mathrm{m}$.

\section{OUTSIDE CYLINDER RIBBON LAYING}

\section{A. Use of a Coordinate Measuring Machine}

Using a large-volume Coordinate Measuring Machine (CMM) to mount ribbons precisely has proven successful because of the high level of quality control available. The CMM provides continual feedback of information that insures precise mechanical alignment throughout the entire mounting operation. The CMM is not just used to verify ribbon position after placement. Among the tasks performed are the following:

- Precise inspection and characterization of ribbons and cylinders prior to ribbon mounting.

- Control ribbon placement and verification of mechanical alignment during all steps of ribbon mounting.

- Immediate verification of a ribbon's position afterward by sampling fibers in the ribbon.

- Immediate calibration of the detector by measurement of actual fiber locations. Achieving spatial calibration prior to installation can be a significant advantage for early analysis of data.

We have chosen to use a large bridge-type CMM with absolute measuring accuracy of 0.0005 inch bandwidth (per B89 standard) over a volume of $3251 \mathrm{~mm}$ by $1829 \mathrm{~mm}$ by $1372 \mathrm{~mm}$.

\section{B. Cylindrical Support}

The ribbons are mounted on a lightweight but rigid cylinder with the cross sectional structure shown in Figure 3. It is constructed from a honeycomb paper core sandwiched between composite layers consisting of 4 thin veneers with a total thickness of 0.010 inch of carbon fiber and epoxy resin. This composite structure results in an effective mass of only $0.5 \%$ radiation lengths at $90^{\circ}$ to the beam line. The variation of both inner and outer surfaces from a prefect cylinder has been measured to be $\sigma<100 \mu \mathrm{m}$ for points over the entire surface and at $\sigma=20 \mu \mathrm{m}$ locally. This surface precision will allow ribbons widths of up to 400 hundred channels to be placed on it without compromising the measured spatial resolution.

\section{Prototype Outside Cylinder Ribbon Laying Machine}

The outside cylinder ribbon mounting process is facilimted mechanically by a ribbon laying machine, shown in Figure 4. The cylinder is attached via end plates fixed to a steel shaft. This shaft is supported at both ends by bearings mounted to a rigid steel framework. Brakes applied at both ends of the shaft fix the cylinder in a given angular location. Above the cylinder, a precision jig plate mounted on linear track sliders is used to hold the ribbon while mounting. This jig plate was machined from $1 / 4$ inch plate stock of 7075 aluminum alloy, in a highly accurate manner similar to the jig plates used for ribbon production. v-shaped grooves for positioning the fibers are cut along the length of the plate at precise intervals to match the fiber pitch. We compensated for the distortion induced in the plate as it was bolted to supports on the machine by adding shim stock under and behind the plate. A straightness of better than $20 \mu \mathrm{m}$ was achieved over the full $2 \mathrm{~m}$ length of the plate by checking down grooves with the CMM.

The linear track sliders to which the plate is attached allow the plate's position to be adjusted horizontally with respect to the cylinder. Micrometer head adjustments and clamps are used to precisely position each end of the plate in an aligned location determined by the CMM.

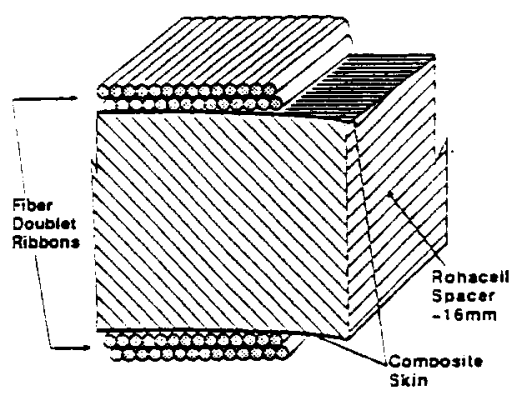

Fig. 3 Cross sectional view of a composite structure for cylindrical ribbon support.

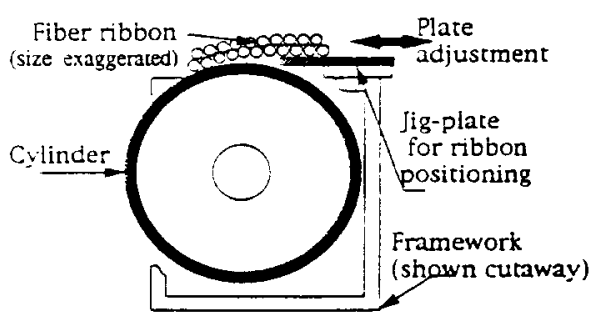

Fig. 4 Features of the prototype ribbon laying machine.

\section{Ribbon Mounting Procedure}

To establish a baseline cylindrical coordinate system for our test, we mapped the cylinder's entire surface under the 
CMM prior to mounting any ribbons. This map was then referenced to tooling balls mounted on the cylinder. Alignment of the ribbons and the cylinder could then be verified at any time during subsequent steps simply by locating the tooling balls and jig plate with the CMM.

After applying a layer of transfer adhesive to the surface of the cylinder, (we used 0.002 0.005 mil-thick adhesive manufactured by $3 \mathrm{M}$ Corporation), the jig plate is guided into alignment by adjusting the micrometer heads. With the aid of the CMM, end-to-end alignment of $20 \mu \mathrm{m}$ can be regularly achieved.

A ribbon is then placed in the jig so that roughly two thirds of its width extends off the plate over the cylinder and only one third is held by the grooves.

The part of the ribbon extending over the cylinder is then allowed to "hinge down" slowly into contact with the cylinder, so that the leading edge fibers (30 or so) touch, defining the ribbon's placement.

When a bond at the edge of the ribbon has been achieved, the other edge of the ribbon is lifted out of the plate and the plate is retracted. The remainder of the ribbon is then allowed to come into full contact with the cylinder. Since some handling of the ribbon is involved, there is the danger of disturbing a ribbon's alignment. We found that after some practice this could be controlled by taking care that lateral stresses were not applied when touching the ribbon.

\section{E. Placement Accuracy}

The placement accuracy achieved for ribbons mounted in both axial and stereo configurations (calibrated for fiber pitch
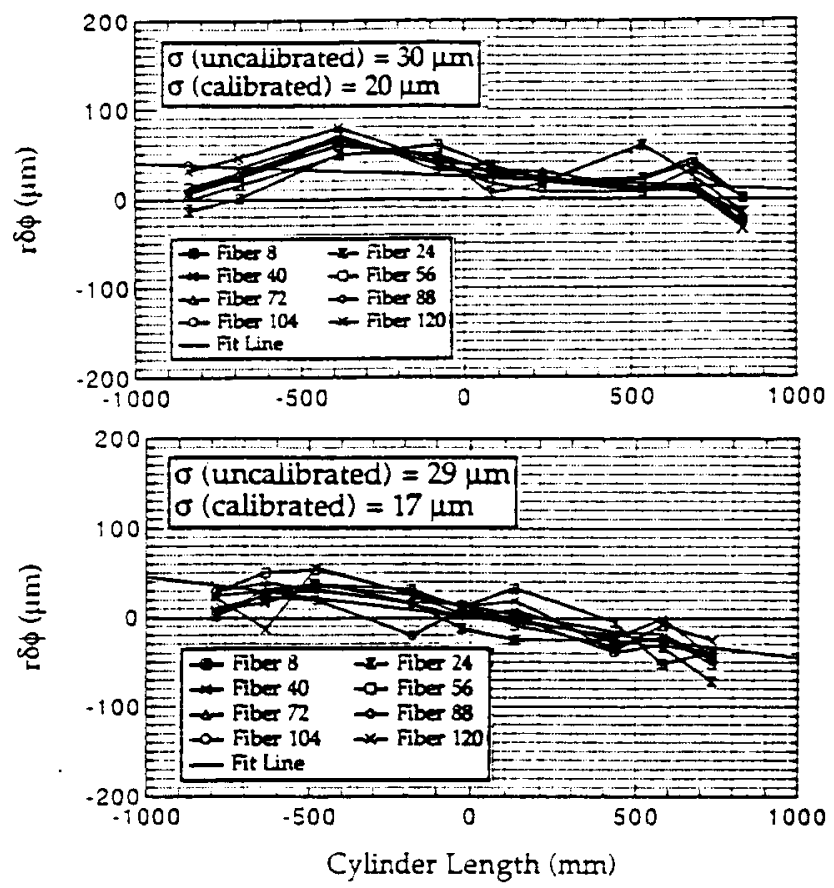

Fig. 5 Measured deviations of fiber positions from a target location in $r \delta \phi$, for ribbon mounted parallel (top) and at $2^{\circ}$ stereo angle (bottom) from the cylinder's axis. only) are plotted in Figure 5 . In both cases, the offset from the target position in $r \delta \phi$ is small, $\sim 30 \mu \mathrm{m}$, and the deviation from alignment along the entire $2 \mathrm{~m}$ length is also $-30 \mu \mathrm{m}$. By calibrating each ribbon for the offset and slope, represented by the fit lines in Figure 5, the fiber position can be known to $\sigma(r \delta \phi)=20 \mu \mathrm{m}$. This is essentially the intrinsic accuracy of the fiber alignment within the ribbons themselves. Deviations of the fiber positioning in the direction of the cylinder's radius were also measured to be consistent with the fluctuations in the cylinder surface's shape $<100 \mu \mathrm{m}$, even when multiple layers of doublets were built up into a superlayer.

In conclusion, CMM control results in a precision method for ribbon placement. CMM measurements provide us with a powerful means by which we can make an immediate calibration for fiber alignment prior to installing a tracker in an experimental setup. Because of the accuracy in ribbon construction and placement, only a few calibration constants per multi-channel ribbon are needed to reach the best possible spatial resolution in a large fiber tracking system.

\section{INSIDE CYLINDER RIBBON LAYING.}

\section{A. The Prototype "Inside Cylinder Ribbon Laying Machine"}

The essential features of an "Inside Cylinder Ribbon Laying Machine" (ICRLM) machine, shown in Figure 6, are: (1) a "vacuum" jig-plate for aligning the ribbon along the cylinder axis and holding the ribbon "upside -down" during

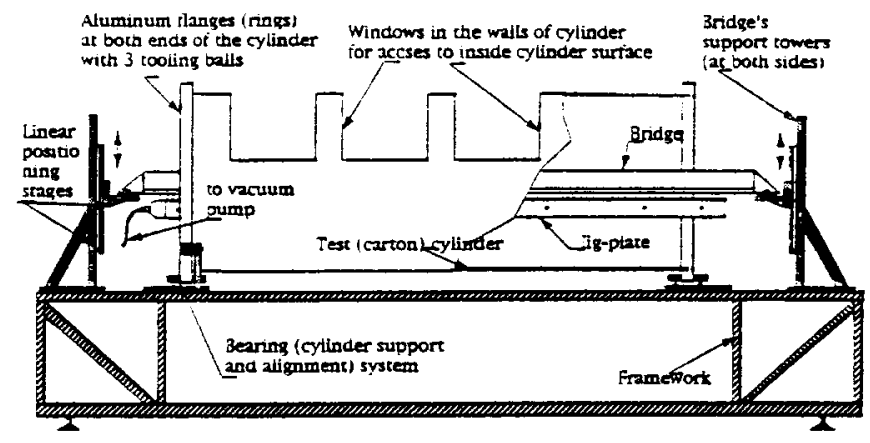

Fig. 6 Features of prototype inside cylinder ribbon laying machine.

the laying procedure; (2) an aluminum box beam bridge (Fig. 2) from which the jig-plate was hung; (3) two support towers onto which the bridge was loaded; (4) linear positioning stages (with micrometer head adjustment) at both support towers for accurately positioning the jig-plate in horizontal and vertical directions; (5) cylinder bearing system, which allows the alignment, rotation and fixing of the test cylinder in a selected position; and (6) a steel framework for rigid support of the bearing system, the test cylinder, and the bridge's support towers.

In building our prototype, we used a hard board (paper) cylinder with an intemal radius of $255 \mathrm{~mm}$, a length of $2 \mathrm{~m}$. 
Both ends of this cylinder are glued to aluminum flanges (rings), which allow the cylinder to be loaded into the bearing system. Each ring has six tooling balls, three of which were used as reference points throughout our analysis.

In order to determine the quality of the internal surface of our test cylinder, we measured 200 points on the inside surface using the CMM. Although the precision of the hard board test cylinder $(\delta \mathrm{r}<160 \mu \mathrm{m})$ is far from the surface precision we obtained with our carbon fiber composite test cylinders [2], it is adequate for testing inside cylinder mounting techniques.

The vacuum jig-plate, a cross-section of which is shown in Figure 7, is an important part of the mechanical setup. Its dimensions are $2.2 \mathrm{~m}$ in length by $12 \mathrm{~cm}$ by $16 \mathrm{~cm}$. The jig plate was constructed of two aluminum box beams placed between two aluminum plates at the top and bottom. This configuration forms a rigid yet light weight system with three separate vacuum chambers. In order to keep internal thermal stresses to a minimum, the parts were assembled using glue and screws rather than by welding. The bottom plate, used for ribbon alignment, was machined from $1 / 2$ " plate stock of 7075 alloy aluminum, in a manner similar to that of jig-plates used in ribbon production [4]. The v-shaped grooves for positioning the fibers were cut along the length of the plate at $870 \mu \mathrm{m}$ intervals or pitch to match the fiber spacing in our test ribbons. The deviation from straightness measured for this jig is approximately $7 \mu \mathrm{m}$.

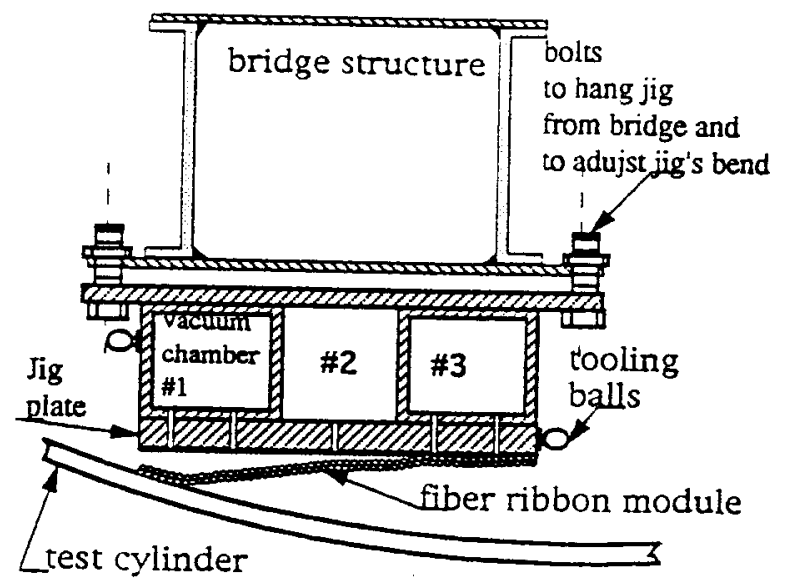

Fig. 7 Cross section of bridge and vacuum jig-plate.

Seven tooling balls were mounted along each side of the jig-plate. By referencing the locations of the v-grooves relative to the tooling balls, we could quickly determine the position of the fiber ribbon during the placement procedure.

Holes through the bottom jig-plate connecting to the vacuum chambers held the ribbon in position against gravity. For our test, we chose holes $3 \mathrm{~mm}$ in diameter, and used a simple vacuum system consisting of a vacuum pump, a reservoir tank, plastic tubes with shut off valves, and gages. This vacuum system allowed the independent release of vacuum from each of the three chambers. The jig-plate was not flat initially. It "bowed" from internal stress such that its center was about $600 \mu \mathrm{m}$ above the ends. This effect and any gravitational sag were compensated for by connecting the jig to a bridge using 14 fine thread alignment bolts. These bridge hangers were adjusted in order to make the surface flat. The bridge was made as a light box-beam structure by welding aluminum plates and I-beams (See Figure 7). After adjustment vertical bend was less than $60 \mu \mathrm{m}$ : more than acceptable for accurate ribbon placement.

Loading struts extend from both ends of the bridge. These allow the bridge to be loaded onto the support towers. Linear positioning stages, with micrometer head adjustment, on both bridges' support towers are used to position the jig in the horizontal and vertical directions in precise alignment above the cylinder's surface.

For structural rigidity, the machine's framework was constructed of steel box beams supported off the floor by adjustable feet.

\section{B. Inside Cylinder Ribbon Mounting Procedure}

Prior to the mounting procedure, the CMM measured the plate's tooling balls and several grooves on the jig-plate at 10 points along each groove. CMM measurements and software analysis were used to determine the positions of the plate's grooves, and accordingly the location of the ribbon, relative to the tooling balls. Therefore, during all following steps only measurements of the plate's tooling balls were required.

(1) The test cylinder is loaded into the bearing system with the "windows" facing up, then the brakes are applied. In this position, the CMM probe has access to the inside cylinder surface, see Figure 7.

(2) The CMM measures the tooling balls mounted to the cylinder's aluminum rings to find the cylinder's exact orientation. The CMM coordinate system is then aligned exactly with the cylinder's axis and rotation angle by on-line software.

(3) With the bridge-jig plate system outside of the cylinder, a ribbon is placed onto the jig plate. Before placing it on the vacuum jig-plate, the ribbon was clamped on a production jig plate for about 4 hours to assure the set of the proper pitch [2]. The vacuum pump is then turned on to pump out the vacuum chambers, securing the ribbon to the jig. The jigbridge system is then loaded onto the support towers inside the cylinder.

A strip of 2 mill thick transfer adhesive (3M) is applied to the ribbon and smoothed down. Typically twenty minutes is allowed for the adhesive to adhere before the backing is carefully peeled off. The layer of adhesive seals the ribbon well, allowing pressure differences of $10 \mathrm{psi}$ in the chambers. This vacuum creates enough force to keep the ribbons in the grooves without shifting when the jig is umed over.

(4) Once the bridge's struts are loaded onto the support towers, the CMM measures the positions of tooling balls, at each edge of the plate. Those tooling balls are located outside of the cylinder's volume, and so are within reach of the CMM's probe. The on-line software then indicates the required adjustments in order for the ribbon to reach its desired 
location. The jig-plate is then guided by linear positioning stages using micrometer head adjustments into precise position very close to surface of cylinder. The distance between the surface of the cylinder and the edge of the ribbon in the "drop position" is approximately $1 \mathrm{~mm}$.

(5) When all is ready, the vacuum in chamber \#1 is released and the left edge of the ribbon is allowed to drop slowly into contact with the cylinder (Figure 7). At this point, about 5 to 10 fibers are touching the cylinder's surface. After twenty minutes, the vacuum in chamber $\# 2$ is released. The ribbon is flexible enough to curve in order to match the cylinder's surface. At this point about $1 / 3$ of the ribbon, about 40 fibers, is touching the cylinder's surface. About $1 / 3$ of the ribbon is still located in the jig's grooves. Twenty minutes later, we release the vacuum in the final chamber and the ribbon comes completely into contact with the cylinder.

(6) Finally, the ribbon is qualified by CMM measurement. Even without windows in a cylinder, the CMM should be able to measure fibers near the open ends of the cylinder. Since the ribbons lay straight on the cylinder surface, this should be adequate to calibrate a ribbon's location .

\section{Inside Cylinder Placement Accuracy}

After placing a ribbon using the above procedure, we measured along two fibers in the ribbon. In order to ensure that each fiber was measured correctly, the fiber was chosen then measured at each end, giving two points. The CMM probe then measure along the line connecting those two
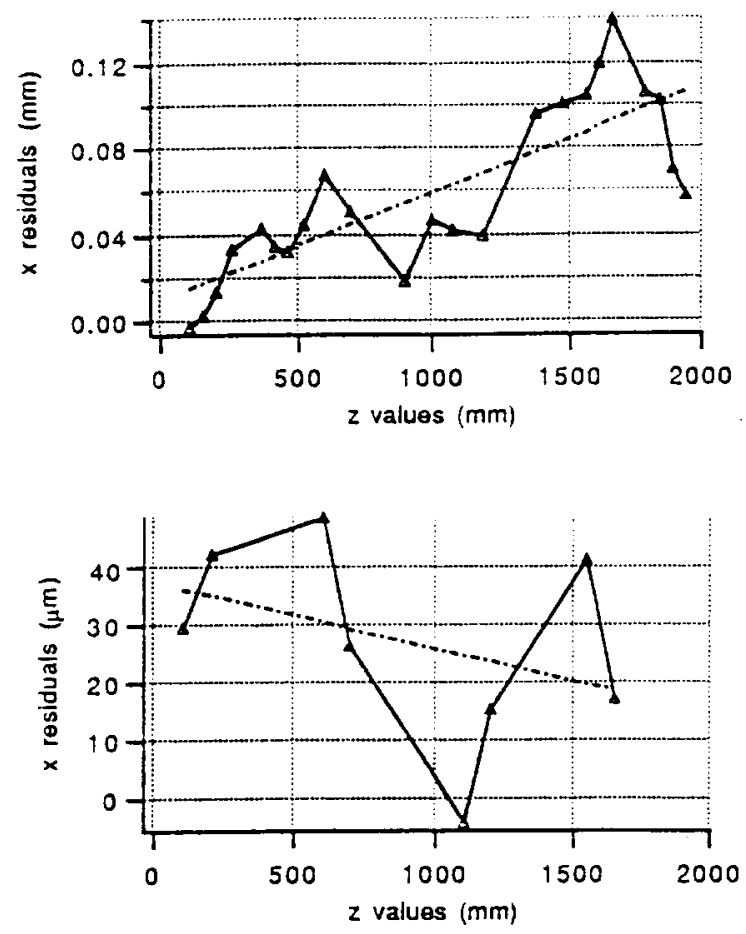

Fig. 8 The solid line gives the difference between data and prediction, and dashed line is the best fit, for $x$ values of the 48 th (top) and 54th (bottom) fibers.
points.For example, Figure 8 (top plot) shows that for the 48th fiber, we placed this fiber to within an accuracy of better than $50 \mathrm{~mm}$ per meter. For fiber 54 (Fig.8 (bottom plot)), the results appear to be even better (less than $12 \mu \mathrm{m}$ per meter). The average missalignment is $<35 \mu \mathrm{m}$. However, we know from our measurements of the jig plate that the fibers should be parallel, and thus equally misaligned with respect to our intended axis. The difference between the two fibers therefore indicates that a small error must have been introduced in our analysis due to the unstable nature of the hard board test cylinder. However we can use our worst result to place an upper limit on our misalignment of $50 \mu \mathrm{m}$ per meter for inside ribbon laying. From this results we are satisfied that we have an excellent understanding of scintillating fiber placement on the inside and outside of a cylindrical surface.

\section{CONCLUSION.}

These techniques that we have developed for the accurate placement of scintillating fibers on the inside and outside of a cylindrical surface has proven successful by meeting our design goals. We have built a number of prototype detectors that have undergone test in the Lab 6 Cosmic Ray test stand at Fermilab[3]. These tests have verified the technique resulting in observed spatial resolutions of $92 \mu \mathrm{m}$ for 3000 channel scintillating fiber prototype tracking system[4].

\section{ACKNOWLEDGMENTS}

We thank, Purdue University Shops for their precision machining and Fermilabs Scintillating Fiber Tracking Group at D0 for their generous support.

\section{REFERENCES}

[1]. D.Koltick, "Scintillating Fiber Charged Particle Tracking at the SSC", in Proceedings of Workshop on Scintillating Fiber Detector, Notre Dame, Indiana, October 1993, pp. $111-125$.

[2]. B. Howell, "A Precision Technique for Mounting Scintillating Fiber Ribbons on a Cylinder for Charged Particle Tracking", in Proceedings of Workshop on Scintillating Fiber Detector, Notre Dame, Indiana, October 1993, pp. 515-524.

[3]. Y. M. Park, " Cosmis Ray Test Results of the Do Prototype Scintillating Fiber Tracker", Nuclear Physics B (Proc. Suppl.), 44(1995), pp.332-339

[4]. See M.R.Wayne in this proceeding. 